\title{
Potencial de biossorção do zinco pela macrófita egeria densa
}

\author{
Biosorption potential of zinc by egeria densa macrophytes
}

\begin{abstract}
Aparecido Nivaldo Módenes
Engenheiro Químico pela Universidade Estadual de Maringá (UEM). Doutor em Engenheira Química pela Universidade Estadual de Campinas (Unicamp). Coordenador e professor do Programa de Pós-Graduação em Engenharia Química pela Universidade Estadual do Oeste do Paraná (Unioeste)
\end{abstract}

\author{
Juliana Martins Teixeira de Abreu Pietrobelli \\ Engenheira Química pela Unioeste. Mestre em Engenheira Química pela Unioeste
}

Fernando Rodolfo Espinoza Quiñones

Físico pela Universidad Nacional de Ingenieria. Doutor em Física pela Universidade de São Paulo (USP). Professor do Programa de Pós-Graduação em

Engenharia Química pela Unioeste

\section{Pedro Yahico Ramos Suzaki}

Acadêmico de graduação de Engenheira Química pela Unioeste

\section{Vanessa Lizeria Alflen}

Acadêmica de graduação de Engenheira Química pela Unioeste

\begin{abstract}
Márcia Regina da Silva Fagundes Klen
Química Industrial pela Universidade Federal de Santa Maria (UFSM). Doutora em Engenheira Química pela UEM. Professora do Programa de Pós-Graduação em Engenharia Química pela Unioeste
\end{abstract}

\section{Resumo}

Neste trabalho, foi estudada a capacidade de biossorção da macrófita aquática Egeria densa seca na remoção do íon zinco. Os testes cinético e de equilíbrio foram realizados em sistema de batelada com temperatura, agitação e pH controlados. Os resultados obtidos na cinética de biossorção demonstram uma rápida remoção do zinco (II), com tempo de equilíbrio em torno de 45 minutos e taxa de remoção superior a 70\%. O modelo cinético de pseudo-segunda ordem foi o que melhor representou os dados experimentais. No estudo do equilíbrio, verificou-se que o modelo de Langmuir ajustou-se melhor aos dados experimentais, com capacidade máxima de sorção de 0,92 mequiv $\mathrm{g}^{-1}$ e razão entre as taxas de sorção e dessorção de $0,829 \mathrm{~L} \mathrm{~g}{ }^{-1} \mathrm{em}$ pH 5 . Os resultados obtidos demonstram que a macrófita $E$. densa tem potencial para ser utilizada em sistemas de tratamento de efluentes industriais.

Palavras-chave: Biossorção, Egeria densa, Zn (II).

\begin{abstract}
In this paper, the removal potential on $\mathrm{Zn}$ ion by macrophyte Egeria densa has been studied. The influence of the metal solution pH, the plant drying and the metal solution temperature, and biosorbent grain size was previously studied in batch systems. Adsorption kinetic and equilibrium experiments of metals onto E. densa were performed under controlled temperature and permanent shaking. In adsorption kinetic tests for Zn (II) the equilibrium time was around 45 min. The biosorption kinetic data were well fitted by a pseudo-second order model. The equilibrium data at pH 5 were described a rather better by the Langmuir isotherm than the Freundlich one, with an adsorption rate and maximum metal content values of 0,829 $\mathrm{g}^{-1} \mathrm{and}_{0,92}$ mequiv $\mathrm{g}^{-1}$, respectively, for Langmuir model. The macrophytes $E$. densa could be used as biosorbent material in industrial effluent treatment system.
\end{abstract}

Keywords: Biosorption, Egeria densa, Zn (II). 


\section{Introdução}

O uso indiscriminado dos recursos naturais tem causado sérios problemas de contaminação de lençóis freáticos e cursos de água, principalmente devido à poluição química de origem orgânica e inorgânica. O zinco é um dos poluentes mais comumente encontrados nos efluentes industriais. As emissões antropogênicas são maiores que as naturais, provenientes das metalúrgicas, bacias de rejeitos da mineração, cinzas de processos de combustão, e o uso de produtos comerciais, tais como conservantes de madeira e fertilizantes a base de zinco (CLARISSE; AMORIM; LUCAS, 1999).

Vários são os métodos convencionais de tratamento disponíveis para a remoção de metais pesados dissolvidos em efluentes líquidos, podendo-se citar: precipitação, oxidação e redução química, ultrafiltração, eletrodiálise e osmose reversa, processos eletroquímicos, troca iônica e evaporação (OZCAN et al, 2005) e processos de sorção (DAVIS; VOLESKY; MUCCI, 2003; MARTÍNEZ et al, 2006). Entretanto, quando estes métodos são aplicados em efluentes com concentrações baixas de metais, podem haver custos elevados, baixa eficiência da remoção, baixa seletividade e alto requerimento de energia.

O processo da biossorção que emprega como adsorvente materiais de origem natural surge como uma tecnologia promissora e em atual expansão em relação à sua aplicação no tratamento e polimento final de efluentes contendo metais pesados (KRATOCHVIL; VOLESKY, 1998).

Diferentes materiais biológicos vêm sendo testados na remoção do zinco, tais como bactérias (CHEN et al, 2009), algas (CRUZ et al, 2004; SENTHILKUMAR et al, 2006; FAGUNDES-KLEN et al, 2007), fungos (BAYRAMOGLU; YAKUP ARICA, 2008) e plantas (UCUN; AKSAKAL; YILDIZ, 2008; CHAKRAVARTY et al, 2007).

Dentre os diversos materiais utilizados como biomassa, as macrófitas têm se destacado por sua eficiência na remoção de íons metálicos (RUBIO; SCHNEIDER, 2003). Apesar de diversas macrófitas terem sido testadas como biossorventes, há poucos estudos sobre a aplicação da E. densa, considerada a principal planta daninha em diversas partes do mundo (BINI; THOMAZ, 2005). A E. densa (elodea brasileira) é uma macrófita aquática de ambiente limnético, submersa, enraizada, nativa da América do Sul.

Desta forma, este trabalho tem como objetivo estudar a remoção do íon zinco utilizando a biomassa seca da macrófita E. densa por meio dos testes cinéticos e de equilíbrio.

\section{Metodologia}

\section{Preparo da biomassa}

A macrófita aquática E. densa foi coletada no Centro de Pesquisas Avançadas em Aquicultura (CPAA), na cidade de
Toledo (PR), lavada com água destilada, sendo uma parte seca em temperatura ambiente $\left(30^{\circ} \mathrm{C}\right)$ e outra em estufa $\left(50^{\circ} \mathrm{C}\right)$. Após este processo, as macrófitas foram trituradas para utilização nos experimentos

\section{Soluções de zinco e AAS}

Para a realização dos experimentos em batelada, foram preparadas soluções de zinco com aproximadamente 4 mequiv $L^{-1}$ (dissolução em água deionizada) a partir de cloreto de zinco puro $\left(\mathrm{ZnCl}_{2}\right)$. Para o ajuste de $\mathrm{pH}$, foram utilizadas soluções de $\mathrm{NaOH}(1 \mathrm{M})$ e $\mathrm{HCl}(1 \mathrm{M})$.

As concentrações do íon zinco foram medidas utilizando-se um espectrômetro de absorção atômica (EAA), modelo AA 932-GBC.

\section{Testes preliminares}

Para a obtenção do limiar de pH em que se inicia a precipitação, fenômeno que pode interferir na avaliação do processo de biossorção, foi utilizada uma solução com concentração inicial de 4,35 mequiv $\mathrm{L}^{-1}$ de zinco, distribuída em alíquotas com $\mathrm{pH}$ ajustado na faixa de 3 a 8 . O teste de precipitação do metal foi realizado em duplicata, sem agitação, em temperatura ambiente $\left(30^{\circ} \mathrm{C}\right)$ por 48 horas. O precipitado foi separado da solução por meio de um sistema de filtração a vácuo utilizando-se membranas com tamanho de poros de $0.45 \mu \mathrm{m}$ e a concentração do zinco medida por EAA.

A influência da temperatura na secagem da biomassa na biossorção foi avaliada utilizando $250 \mathrm{mg}$ de E. densa, seca em temperatura ambiente $\left(30^{\circ} \mathrm{C}\right)$ e em estufa $\left(50^{\circ} \mathrm{C}\right)$, em contato com $50 \mathrm{~mL}$ de solução em erlenmeyer de $125 \mathrm{~mL}$. Todos os testes foram realizados em duplicata com concentração inicial de 4,15 mequiv $\mathrm{L}^{-1}$ de zinco, mantidos sob agitação em "shaker" a $30^{\circ} \mathrm{C}$, por 12 horas. $\mathrm{O}$ zinco residual da solução foi separado do biossorvente através de um sistema de filtração a vácuo e sua concentração medida por EAA.

$O$ efeito da temperatura da solução foi avaliado variando a temperatura em intervalos de $5^{\circ} \mathrm{C}$, na faixa de 25 a $45^{\circ} \mathrm{C}$, com a planta seca a $30^{\circ} \mathrm{C}$. O efeito do tamanho das partículas do biossorvente foi avaliado utilizando-se partículas de macrófitas com granulometria de 0,589, 0,295 e 0,147 mm e sem separação. Este teste foi conduzido em temperatura de $30^{\circ} \mathrm{C}$ e com concentração inicial de $\mathrm{Zn}$ igual a 3,77 mequiv $\mathrm{L}^{-1}$. Todos os testes foram realizados em duplicatas.

\section{Teste cinético}

Neste teste, também realizado em duplicata, a temperatura foi controlada em $30^{\circ} \mathrm{C}$, sob agitação constante e em pH 5. Em intervalos pré-definidos foram retiradas amostras, filtradas e a concentração do zinco medida por EAA. 


\section{Estudo de equilíbrio}

Em duplicata, este teste foi realizado em frascos erlenmeyer de $125 \mathrm{~mL}$ contendo $50 \mathrm{~mL}$ de solução do íon metálico, adicionaramse de 20 a $550 \mathrm{mg}$ de biomassa. Os testes foram realizados em pH 4 e 5 , sob agitação por 12 horas e temperatura controlada de $30^{\circ} \mathrm{C}$. Ao final de cada ensaio, a fase líquida foi separada do biossorvente por meio de filtração em membrana de 0,45 $\mu \mathrm{m}$. As concentrações iniciais e de equilíbrio de zinco para cada amostra foram determinadas por EAA.

\section{Resultados e Discussão}

\section{Testes preliminares}

Analisando-se o resultado do teste de precipitação do íon zinco apresentado na Figura 1, verifica-se que a precipitação do zinco ocorre para valores de $\mathrm{pH}$ maiores que 5,5. Assim, todos os ensaios de biossorção foram realizados em valores de $\mathrm{pH}$ abaixo de 5 , minimizando erros de quantificação quanto à remoção de metal pela biomassa utilizada.

No teste da influência da temperatura de secagem da biomassa, obteve-se uma remoção do zinco superior a 70\% para biomassa seca em temperatura ambiente $\left(30^{\circ} \mathrm{C}\right)$, enquanto para biomassa seca em estufa $\left(50^{\circ} \mathrm{C}\right)$ a remoção do zinco foi em torno de $55 \%$. Verificouse, portanto, uma taxa de remoção significativamente maior para a temperatura de $30^{\circ} \mathrm{C}$.

Os resultados obtidos no teste do efeito da temperatura da solução mostraram que a maior taxa de remoção, cerca de $70 \%$, foi alcançada em $30^{\circ} \mathrm{C}$.

No teste da influência da granulometria na biosorção, para os tamanhos de partículas de biomassa testados, verificou-se que a diferença entre a taxa de remoção de zinco foi menor que $2 \%$ em relação à taxa sem separação, podendo ser desprezada por estar dentro da margem do erro experimental.

Assim, para o teste cinético e no estudo do equilíbrio de sorção, foi utilizada a biomassa sem separação granulométrica seca em temperatura ambiente. A temperatura da solução foi de $30^{\circ} \mathrm{C}$.

\section{Teste cinético}

A Figura 2 apresenta os resultados do teste cinético da biossorção do zinco pela biomassa da macrófita E. densa. Verificou-se uma redução de 50\% na concentração de zinco nos primeiros 10 minutos, atingindo o equilíbrio em torno de 45 minutos. Este comportamento com sorção inicial rápida seguida de um período mais lento é típico para a biossorção de metais que não envolvem nenhuma reação de energia, mas com uma interação puramente físico-química entre a biomassa e a solução do metal (CRUZ et al, 2004).

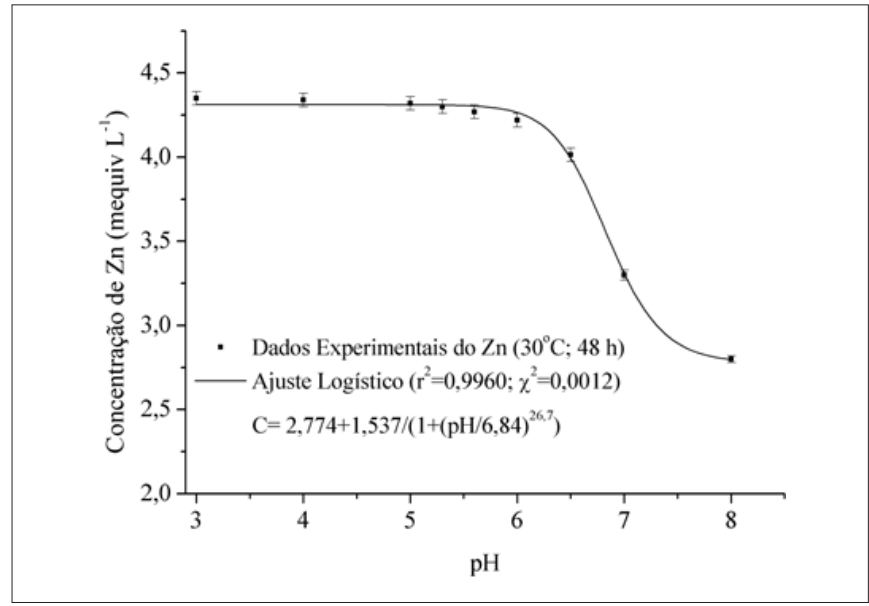

Figura 1 - Concentração do íon Zn (II) em função do pH

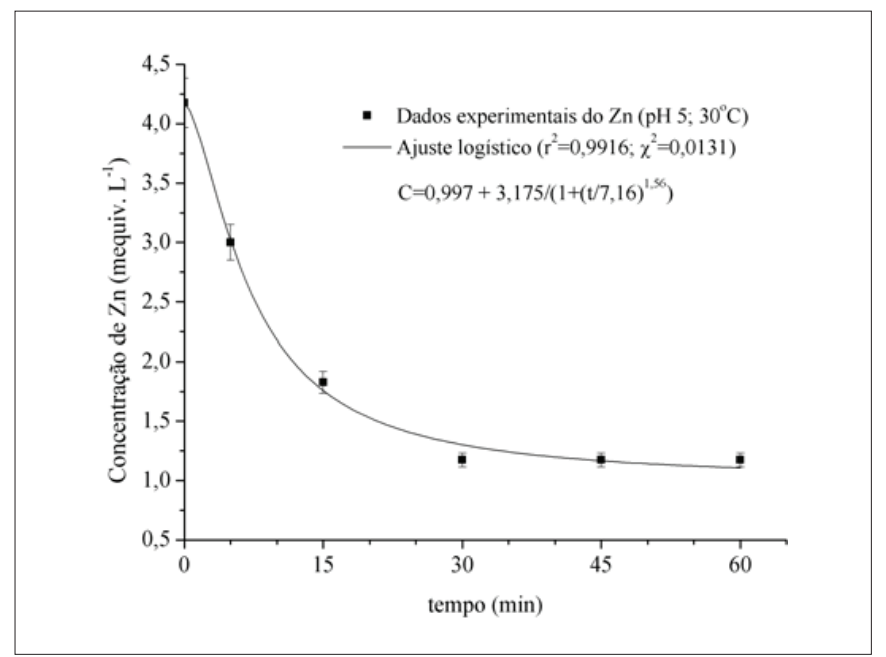

Figura 2 - Cinética da biossorção do zinco (II) pela E. densa

Comparando aos resultados reportados pela literatura, verifica-se que a taxa de remoção de zinco e o tempo de equilíbrio usando a $E$. densa são similares àqueles que utilizamo Pinus sylvestri L. como biosorbente (UCUN et al, 2008) com tempo de equilíbrio de 60 minutos e a biomassa Sargassum filipendula (FAGUNDES-KLEN et al, 2007) com tempo de equilíbrio de duas horas.

\section{Cinética de adsorção}

A concentração de zinco na fase sólida, no tempo t, é calculada usando a Equação 1.

$q_{(t)}=\frac{V\left(C_{0}-C_{t}\right)}{m_{s}}$

Equação 1

$\mathrm{Na}$ qual:

$C_{0}$ : concentração inicial de zinco na solução (mequiv $\mathrm{L}^{-1}$ );

$C_{t}$ : concentração final de zinco na solução;

$V$ : volume da solução (L);

$m_{\mathrm{s}}$ : a massa seca do biossorvente $(\mathrm{g})$. 


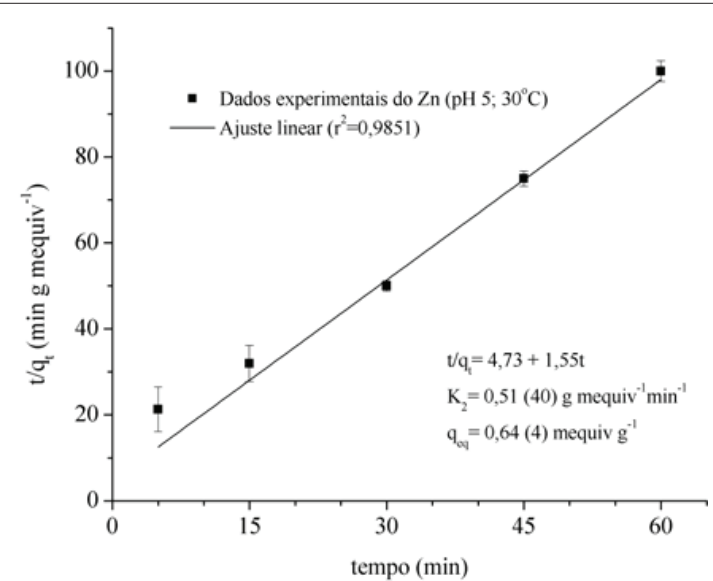

Figura 3 - Dados cinéticos e modelo de pseudo-segunda ordem do processo de biossorção do zinco pela $E$. densa

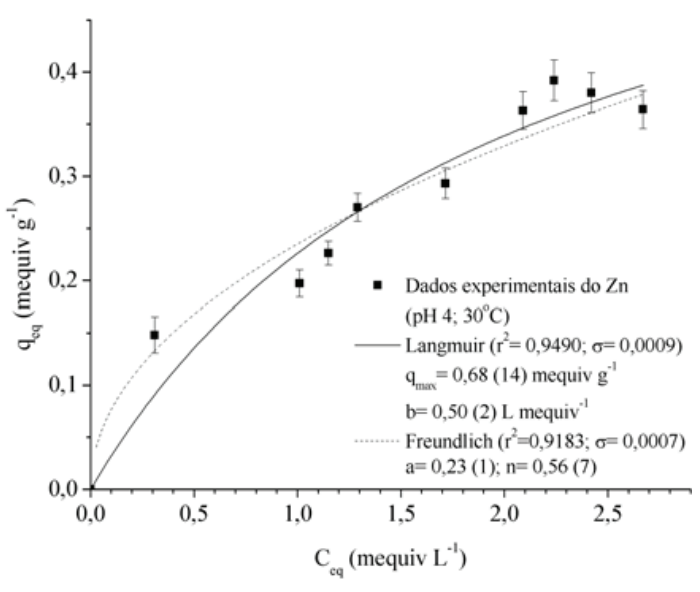

Figura 4 - Dados experimentais de equilíbrio para a biomassa $E$. densa e isotermas de Langmuir e Freundlich para pH 4

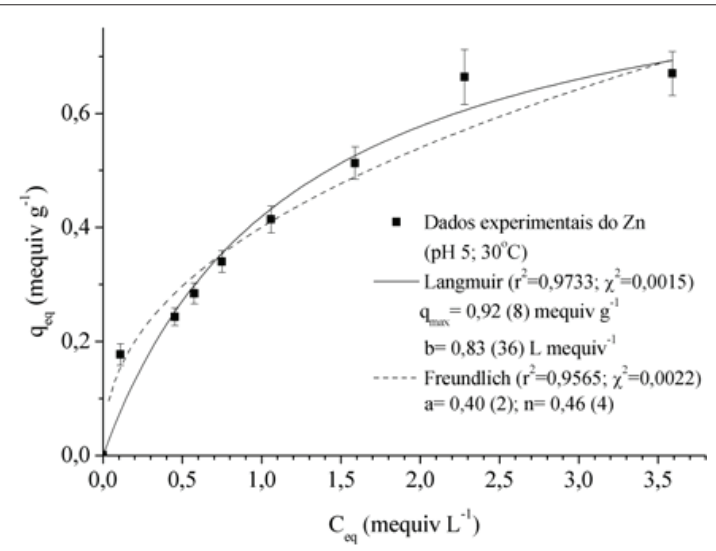

Figura 5 - Dados experimentais de equilíbrio para a biomassa $E$. densa e isotermas de Langmuir e Freundlich para pH 5

Os modelos cinéticos mais usados na biosorção são o pseudoprimeira ordem e o pseudo-segunda ordem (HO; MCKAY, 1998). A forma linear do modelo pseudo-primeira ordem, descrito por Lagergren, é apresentada pela Equação 2. $\log \left(q_{e q}-q_{t}\right)=\log \left(q_{e q}\right)-\left(\frac{K_{1}}{2,303}\right) t$

Equação 2

Na qual:

$\mathrm{K}_{1:}$ constante de velocidade $\left(\mathrm{min}^{-1}\right)$;

$\mathrm{q}_{\mathrm{eq}}$ : quantidade de metal sorvido no equilíbrio (mequiv $\mathrm{g}^{-1}$ );

$\mathrm{q}_{\mathrm{t}}$ : a quantidade de metal sorvido no tempo $\mathrm{t}$ (mequiv $\mathrm{g}^{-1}$ ).

O modelo linear de pseudo-segunda ordem é apresentado pela Equação 3.

$\frac{t}{q_{t}}=\frac{1}{K_{2} q_{e q}^{2}}+\frac{1}{q_{e q}} t$

Equação 3

$\mathrm{Na}$ qual:

$\mathrm{K}_{2}$ : constante de velocidade ( $\mathrm{g}$ mequiv ${ }^{-1} \mathrm{~min}^{-1}$ ).

Dentre esses modelos, o que obteve melhor ajuste aos dados cinéticos foi o de pseudo-segunda ordem, apresentado na Figura 3. Os valores da constante de velocidade e da quantidade adsorvida no equilíbrio são 0,51 $\pm 0,40 \mathrm{~g}$ mequiv $^{-1} \mathrm{~min}^{-1}$ e 0,64 $\pm 0,04$ mequiv $\mathrm{g}^{-1}$, respectivamente.

\section{Isotermas de sorção}

Os modelos mais utilizados no estudo da biosorção são as isotermas de Langmuir e Freundlich, apresentados nas Equações 4 e 5, respectivamente.

$q_{e q}=\frac{q_{\mathrm{max}} b \mathrm{C}_{\mathrm{eq}}}{1+\mathrm{bC}_{\mathrm{eq}}}$

Equação 4

$q_{e q}=a\left(C_{e q}\right)^{n}$

Equação 5

Nas quais:

$\mathrm{q}_{\max }$ : capacidade máxima de sorção do metal por unidade de biomas-

sa (mequiv g-1);

b: razão entre as taxas de sorção e dessorção (L mequiv $\left.{ }^{-1}\right)$;

a: constante adimensional relacionada à capacidade;

n: intensidade de adsorção.

Os dados de equilíbrio, juntamente com as curvas das isotermas de Langmuir e Freundlich para os valores de pH 4 e 5 são apresentados nas Figuras 4 e 5, respectivamente. O ajuste das isotermas aos dados experimentais de biosorção foi feito usando o software Origin ${ }^{\circledR}$ 8.0. Os valores das constantes dos modelos de Langmuir e Freundlich obtidos a partir dos dados de equilíbrio estão apresentados na Tabela 1 .

O modelo de isoterma de Langmuir foi o que se ajustou melhor aos dados de equilíbrio da biossorção do íon zinco, de acordo com os valores da correlação e variância, mostrados na Tabela 1. Verifica-se que quanto maior o pH da solução, maior a taxa de adsorção.

Resultados similares de biossorção do íon zinco também são reportados na literatura. Utilizando a biomassa da alga verde Ulva reticulata, Senthilkumar et al (2006) obtiveram os valores 
de $\mathrm{q}_{\max }=26,39 \mathrm{mg} \mathrm{g}^{-1}$ e $\mathrm{b}=0,02 \mathrm{Lg}^{-1}$ para o modelo de Langmuir. Utilizando a biomassa da Sargassum filipendula pré-tratada, Fagundes-Klen et al (2007) encontraram os valores de $\mathrm{q}_{\max }=1,28$ mequiv g-1 e b=6,10 Lmequiv $^{-1}$. Chakravarty et al (2007) também obtiveram valores para os parâmetros de Langmuir muito próximos aos obtidos neste trabalho $\left(q_{\max }=26,31 \mathrm{mg} \mathrm{g}^{-1} \mathrm{e} b=0,62\right.$ $\mathrm{Lg}^{-1}$ ) com a utilização da biomassa de polpa de papel pré-tratada. Em trabalho prévio, Módenes, Pietrobelli e Espinoza-Quiñones (2009) utilizando a E. densa na adsorção do íon cádmio, obtiveram resultados similares aos obtidos na adsorção do íon zinco, com os valores dos parâmetros $\mathrm{q}_{\max }=1.28$ mequiv $\mathrm{g}^{-1} \mathrm{e} b=0.40$ Lmequiv $^{-1}$.

Devido ao baixo tempo de equilíbrio (45 minutos), rápida e alta taxa de remoção do zinco, a macrófita aquática E. densa apresenta grande potencial adsorvente para ser aplicada em sistemas de tratamento de efluentes.

\section{Conclusões}

A partir dos resultados dos testes preliminares pode-se concluir que:

- $\mathrm{O}$ pH ao qual se inicia a precipitação do zinco é superior a 5,5;

- A maior percentagem de remoção foi obtida com a biomassa seca em temperatura ambiente;

- A máxima remoção de zinco foi obtida com a temperatura da solução de $30^{\circ} \mathrm{C}$;
Tabela 1 - Análise estatística e ajuste dos parâmetros das isotermas de Langmuir e Freundlich para o íon zinco

\begin{tabular}{l|ccc} 
Modelos & Parâmetros & $\mathrm{pH} \mathrm{4}$ & $\mathrm{pH} \mathrm{5}$ \\
\hline Langmuir & $\mathrm{q}_{\max }$ & $0,68(14)$ & $0,92(8)$ \\
& $\mathrm{b}$ & $0,50(2)$ & $0,83(36)$ \\
\hline \multirow{3}{*}{ Freundlich } & $\mathrm{r}^{2}$ & 0,9490 & 0,9733 \\
& $\sigma$ & 0,0009 & 0,0015 \\
& $\mathrm{a}$ & $0,23(1)$ & $0,40(2)$ \\
& $\mathrm{n}$ & $0,56(7)$ & $0,46(4)$ \\
& $\mathrm{r}^{2}$ & 0,9183 & 0,9565 \\
\hline & $\sigma$ & 0,0007 & 0,0017 \\
\hline
\end{tabular}

- O tamanho das partículas da biomassa não exerce influência significativa na taxa de remoção do zinco.

A biomassa da macrófita E. densa possui boas características adsorventes, tais como baixo tempo de equilíbrio, capacidade máxima de remoção similar ou superior a outros biossorventes e disponibilidade natural em grandes quantidades em várias regiões. Sendo assim, a E. densa mostra-se um biossorvente eficiente em sistemas de tratamento de efluentes baseados em processos de biossorção.

\section{Agradecimentos}

À Fundação Araucária pelo apoio financeiro e à Fundação para o Desenvolvimento Científico e Tecnológico (Fundetec) de Cascavel (PR) pelo suporte técnico.

\section{Referências}

BAYRAMOGLU, G.; YAKUP ARICA, M. Removal of heavy mercury(II), cadmium(II) and zinc(II) metal ions by live and heat inactivated Lentinus edodes pellets. Chemical Engineering Journal, v. 143, p. 133-140, 2008.

BINI, L.M.; THOMAZ, S.M. Prediction of Egeria najas and Egeria densa occurrence in a large subtropical reservoir (Itaipu Resrvoir, Brazil Paraguay). Aquatic botany, v. 83, n. 3, p. 227-238, 2005.

CHAKRAVARTY, S. et al. Adsorption of zinc from aqueous solution using chemically treated newspaper pulp, Bioresource Technology, v. 98, n. 16, p. 3136-3141, 2007

CHEN, X. et al. Interaction of Pseudomonas putida CZ1 with clays and ability of the composite to immobilize copper and zinc from solution. Bioresource Technology, v. 100, n. 1, p. 330-337, 2009

CLARISSE, M.D.; AMORIM, M.C.V.; LUCAS, E.F. Despoluição ambiental: uso de polímeros na remoção de metais pesados. Revista de Química Industrial, v. 67, n. 715, p. 16-24, 1999

CRUZ, C.C.V. et al. Kinetic modeling and equilibrium studies during cadmium biosorption by dead Sargassum sp. Biomass. Bioresource Technology, v. 91, n. 33, p. 249-257, 2004
DAVIS, T.A.; VOLESKY, B.; MUCCI, A. A review of the biochemistry of heavy metal biosorption by brown algae. Water Research, v. 37, n. 18, p. 4311-4330, 2003

FAGUNDES-KLEN, M.R. et al. Equilibrium study of the binary mixture of cadmium-zinc ions biosorption by the Sargassum filipendula species using adsorption isotherms models and neural network. Biochemical Engineering Journal, v. 34, n. 22, p. 136-146, 2007

HO, Y.S.; MCKAY, G. Pseudo-second order model for sorption processes. Process Biochemistry, v. 34, n. 5, p. 451-465, 1998.

KRATOCHVIL, D.; VOLESKY, B. Advances in the Biosorption of Heavy Metals. Tibtech, v. 16, p. 291-300, 1998.

MARTÍNEZ, M. et al. Removal of lead(II) and cadmium(II) from aqueous solutions using grape stalk waste. Journal of Hazardous Materials, v. 133 n. 1-3, p. 203-211, 2006

MÓDENES, A.N.; PIETROBELLI, J.M.T.A.; ESPINOZA-QUIÑONES, F.R Cadmium biosorption by non-living aquatic macrophytes Egeria densa. Water Science and Technology, v. 60, n. 2, p. 293-300, 2009. 
OZCAN, A. etal. Determination of the equilibrium, kinetic and thermodynamic parameters of adsorption of copper(II) ions onto seeds of Capsicum annuum. Journal of Hazardous Materials, v. 124, n. 1-3, p. 200-208, 2005

RUBIO, J.; SCHNEIDER, I.A.H. Plantas Aquáticas: Adsorventes Naturais para a Melhoria da Qualidade das Águas. In: XIX PRÊMIO JOVEM CIENTISTA - ÁGUA: FONTE DE VIDA, 2003.
SENTHILKUMAR, R. et al. Seaweeds for the remediation of wastewaters contaminated with zinc(II) ions. Journal of Hazardous Materials, v. 136, n. 3, p. 791-799, 2006

UCUN, H.; AKSAKAL, O.; YILDIZ, E. Copper(II) and zinc(II) biosorption on Pinus sylvestris L. Journal of Hazardous Materials, v. 161, n. 2-3, p. 1040-1045, 2008 